\title{
Questions de méthode
}

Le sport et ses enjeux nationaux et internationaux mis en discours

Paul Aron, Micheline Cambron, Gianni Haver, Marie-Ève Thérenty and François Vallotton

\section{(2) OpenEdition}

\section{Journals}

Electronic version

URL: http://journals.openedition.org/belphegor/851

DOI: 10.4000/belphegor.851

ISSN: 1499-7185

Publisher

LPCM

Electronic reference

Paul Aron, Micheline Cambron, Gianni Haver, Marie-Ève Thérenty and François Vallotton, « Questions de méthode », Belphégor [Online], 15-1 | 2017, Online since 06 July 2017, connection on 01 May 2019 URL : http://journals.openedition.org/belphegor/851 ; DOI : 10.4000/belphegor.851

This text was automatically generated on 1 May 2019.

\section{$\Theta \Theta \Theta \Theta$}

Belphégor est mis à disposition selon les termes de la Licence Creative Commons Attribution - Pas d'Utilisation Commerciale - Pas de Modification 4.0 International. 


\title{
Questions de méthode
}

Le sport et ses enjeux nationaux et internationaux mis en discours

\author{
Paul Aron, Micheline Cambron, Gianni Haver, Marie-Ève Thérenty and \\ François Vallotton
}

\section{Un projet collectif}

1 Les travaux sur la presse ont, ces dernières années, pris une envergure considérable. D'abord issus des travaux sur le dix-huitième siècle et, plus particulièrement, sur la période révolutionnaire et ses entours immédiats, développés principalement par Maurice Mouillaud, Pierre Rétat et Jean Sgard dans une perspective interdisciplinaire encore inédite, ces travaux ont lentement migré vers les études des spécialistes de la littérature du XIX ${ }^{e}$ siècle, surpris de découvrir que les écrivains les plus importants du siècle y avaient inventé des genres dont les critiques et les chercheurs avaient rapidement oublié ce qu'ils devaient à la forme même du journal. Les chercheurs de l'équipe à l'origine de ce numéro sont engagés dans des travaux sur les relations entre presse et littérature. Dans leurs recherches respectives, ils s'appuient sur un postulat selon lequel la presse doit être saisie comme une forme spécifique. Dans cette perspective, les journaux ne constituent pas un matériau inerte, utile à la compréhension de l'histoire de la littérature, mais plutôt un ensemble dynamique de formes tendues entre elles et composant tout à la fois un récit, un laboratoire où se joue la poétique des genres et une interface labile entre les discours. Lire le journal, pour ceux qui ont contribué à ce numéro, c'est lire un monde ${ }^{1}$. Et lire ce monde exige les outils de l'analyse littéraire.

2 Nous nous appuyons également sur un certain nombre de travaux qui donnent à ce numéro sa cohérence théorique : travaux de la sociocritique, travaux définitoires des formes de la presse, réflexions sur les notions d'espace public et de transferts culturels. Nous nous sommes également nourris de travaux de nature historique portant sur le mouvement olympique, sur les Jeux de Berlin et sur la pratique du journalisme durant l'entre-deux-guerres que nous avons partagés. Certaines de nos préoccupations rejoignent celles des spécialistes des sciences de l'information, d'autres celles d'historiens 
intéressés à ce que l'on nomme désormais l'histoire culturelle. Nous occupons donc une position transdisciplinaire, tout en revendiquant la nature littéraire de nos travaux.

3 Le caractère transnational du projet s'appuie sur deux constats. Lors d'un séminaire international sur les presses québécoise et suisse romande qui s'est tenu à Lausanne sous la direction de Daniel Maggetti et Micheline Cambron en 2008, les étudiants et les professeurs qui travaillaient sur chacune des deux presses étaient convaincus de mettre à jour des particularités nationales, voire des idiosyncrasies - l'importance de la "petite patrie », la préoccupation pour le développement d'une littérature nationale, l'ancrage des fictions dans le contemporain et la proximité spatio-temporelle, de même que le développement homologue de certaines formes, comme la page féminine et son courrier ou la multiplication des feuilletons, entre autres. Or les échanges ont finalement révélé la similitude de ces pratiques. Que ce diagnostic inattendu tienne à la prégnance de modèles discursifs et génériques largement mis en circulation dans la presse par-delà les frontières nationales, ou qu'il soit lié à une certaine porosité des pratiques liée à la nature du journal, il invitait à ouvrir les travaux sur la presse à la comparaison afin d'éviter la réduction de l'interprétation des résultats de recherche à des questions «locales». Par ailleurs, depuis 2008, les équipes de Marie-Ève Thérenty et Alain Vaillant (La Civilisation du journal), puis celle de Marie-Ève Thérenty et Guillaume Pinson (Médias 19) ont abondamment travaillé sur la présence de formes transnationales, voire de modèles narratifs transnationaux qui ont largement modelé les imaginaires mais aussi les formes. Le cadre habituellement national des travaux sur la presse est ainsi apparu à de nombreux chercheurs comme insuffisant pour comprendre les phénomènes de transferts culturels et de perméabilité des discours que l'analyse littéraire permet de dégager et de problématiser.

4 Les travaux transnationaux ont jusqu'à maintenant été principalement menés autour de genres journalistiques ou autour de topiques et de thèmes, ou encore dans une perspective historique sur la structuration des discours de presse. Le projet québécois dirigé par Micheline Cambron, qui s'est donné comme objectif d'effectuer des comparaisons entre les journaux montréalais de langues diverses (français, anglais, yiddish et italien) en usant d'une méthode classique de carottage autour de dates précises, invitait à opérer les comparaisons sur une base différente, celle d'événements. Nous avons délibéré pour choisir un événement ayant eu des résonances internationales et qui pourrait être saisi grâce à une sorte de carottage de la presse, sur un ensemble limité de dates, conférant ainsi à notre travail une dimension collégiale sans cesse réactivée lors des rencontres et des séances de travail, en personne ou à distance. Nous avons finalement choisi de travailler sur les JO de Berlin qui se sont tenus en 1936. Ceux-ci nous sont apparus comme un choix propre à permettre à la fois une étude substantielle dans chacun des espaces médiatiques sur lesquels nous travaillons, la presse parisienne, belge, suisse romande, montréalaise - à cause de l'importance de la surface qui leur a été consacrée - et susceptible de permettre un travail comparatif stimulant. De plus, nous savions que le retentissement de cet événement, sa portée médiatique, pouvait être saisi en nous limitant à la période même des JO. En effet, dans le contexte de l'étude de la presse, la durée sur laquelle doit s'étendre le dépouillement constitue un enjeu méthodologique majeur et, éventuellement un frein mis à la rigueur de l'interprétation. Par ailleurs, le caractère international de l'événement, qui a été couvert par plus de 1200 journalistes accrédités, et sa dimension hybride - l'événement est sportif, mais aussi politique, diplomatique et culturel, et il sera décliné dans un spectre médiatique très 
large quant aux genres et aux supports - nous sont apparus comme particulièrement stimulants.

\section{Des préoccupations communes...}

5 Notre objectif n'était pas d'abord d'élaborer de nouveaux savoirs, qui s'ajouteraient à ceux qui existent déjà à propos des JO de Berlin, mais plutôt d'analyser la manière dont cet événement avait été couvert dans nos espaces médiatiques respectifs. Nous souhaitions plutôt en étudier la couverture, esquisser une sorte de poétique du journal qui se serait déployée autour de l'événement, voire un ou des imaginaire(s) des JO. Nous postulions que ceux-ci seraient différents selon les pays, les échanges permettant d'affiner les résultats de chacune des équipes et de proposer éventuellement une synthèse dont la nature restait à déterminer. Nous plaçant du côté de la construction du discours social et de ses récits, dans la matière pléthorique de la presse, nous avons également souhaité saisir l'occasion pour développer une réflexion de nature méthodologique sur la presse de l'entre-deux-guerres, sur ses formes et sur le travail lié aux grands corpus de presse du $\mathrm{xx}^{\mathrm{e}}$ siècle réalisés dans une perspective littéraire.

6 Les premières discussions ont fait émerger des attentes, fait surgir des déceptions. $Y$ aurait-il des textes d'auteurs célèbres, des reportages exaltants, des photographies exceptionnelles? Y aurait-il des écrivains-journalistes se découvrant durant les JO ? La couverture sportive serait-elle marquée par les circonstances propres aux Jeux de 1936, menacés de boycott par plusieurs pays, concurrencés par les Jo parallèles de Barcelone? Enfin, quelles traces laissaient dans les divers éléments de la couverture de presse les enjeux idéologiques de la période - racisme et ségrégationnisme institutionnalisé, conflit entre les visions communistes et nazies du monde, la guerre d'Espagne en cours ? La mise en commun des premiers résultats, lors d'une séance de travail conjointe à Montréal, nous a permis de découvrir des variations inattendues - la présence dans les journaux français de clichés très péjoratifs à l'endroit des athlètes asiatiques et des femmes pratiquant l'athlétisme, et leur rareté, voire leur absence ailleurs -, de nous étonner du peu d'allusions faites à la tentative avortée de tenir des JO à Barcelone, de nous interroger sur la question des sources et du recours aux agences de presse, plus cruciale que prévue. La question de la censure des textes des correspondants de presse par le Gouvernement allemand évoquée dans les ouvrages généraux sur les jeux est apparue en outre comme une zone d'ombre de la couverture, quasi absente du discours. Bref, la continuation des travaux a permis d'approfondir la réflexion à partir des particularités décelées dans les divers corpus : pourquoi et comment ces particularités ont-elles pris forme? Quels sont les modèles d'information à l'œuvre dans la couverture des Jo ?

\section{...mais des objets et des outils différents}

7 Les conditions de la recherche ont été fort différentes selon les équipes. Les travaux ont été teintés par les objectifs plus généraux poursuivis par chacune des équipes et par l'état variable des archives journalistiques. Ils ont également été modelés par les outils utilisés, par la nature des dépouillements effectués et par l'existence, ou l'absence, de travaux antérieurs sur la presse de la période et/ou sur la couverture médiatique des sports en général ou des JO de Berlin en particulier, dans un contexte national. Il importe de présenter brièvement les caractéristiques de ces divers processus de recherche. 


\section{L'équipe de Montréal}

8 L'équipe de Montréal était principalement composée de deux chercheuses, Dominique Marquis et Micheline Cambron ${ }^{2}$ et d'un groupe d'assistants de recherche dont le nombre a légèrement fluctué (entre 7 et 9), et qui comptait, au doctorat: Ève Léger-Bélanger, Jérémi Perrault, Ariane Santerre et Marilou St-Pierre ; à la maîtrise Camille Caron Belzile, Xavier Boileau, Alex Giroux, Jeanne Boucher Lauzon et Yosef Robinson. Les dépouillements visaient à la fois à éclairer l'événement choisi, les JO de Berlin, et à engranger des données afin de pouvoir décrire la vie culturelle montréalaise de l'entredeux-guerres telle que les divers périodiques la configurent. Aux fins de ce travail plus ambitieux, l'équipe a élaboré, grâce au soutien d'un chercheur de littérature également informaticien, Olivier Lapointe, une plate-forme qui sert d'interface à une banque de données imaginée et réalisée de manière à épouser la dimension heuristique du travail (identification des champs, ergonomie de l'interface, construction des modes d'interrogation). Perçue comme lourde au début des travaux, l'interface a été régulièrement réajustée et un guide a permis un emploi uniforme des catégories. Elle s'est finalement révélée extraordinairement utile dans le traitement des données liées aux JO de Berlin puisqu'elle permettait de situer chacun des éléments pertinents dans le contexte global des numéros de périodiques dépouillés - et de la couverture sportive. Il faut préciser que la plate-forme permet d'avoir accès, en certains cas, aux pages des journaux que l'équipe a numérisées - la majorité des journaux que nous avons retenus ne sont disponibles que sous forme microfilmée et aucun ne permet une recherche plein texte. Il nous a fallu, au préalable, effectuer un travail de description technique de chacun des périodiques choisis, de manière à pallier le manque d'informations générales sur la presse montréalaise de l'entre-deux-guerres. En effet, outre un ouvrage descriptif général, les rares monographies et articles sur la presse sont principalement centrés sur l'analyse des éditoriaux dans une perspective de dévoilement de leur substrat idéologique : les périodiques de l'entre-deux-guerres québécois ont échappé aux grands chantiers portant sur la presse au Québec ${ }^{3}$. Notre corpus était composé des quotidiens L'Illustration nouvelle, La Patrie, The Montréal Daily Star, Le Canada, Der Keneder Odler, La Presse , The Montreal Herald, Le Devoir et The Gazette, et des hebdomadaires L'Autorité, L'Italia et Le Petit journal. Des journaux dans quatre langues donc.

Deux types de données étaient recueillis dans le cadre d'une analyse systématique de tous les éléments du journal (pas seulement ceux portant sur les Jo donc), depuis les grands titres jusqu'aux entrefilets en passant par la publicité et l'iconographie: des données descriptives liées à la nature de l'élément et à son inscription dans une série (rubrique, chronique, section, page identifiée par un titre et un traitement typographique), à l'emplacement et à la surface occupée, à la source et à la signature, à l'objet traité ; des données linguistiques - temps et modes verbaux, figures de style, expressions saillantes et citations (recueillies dans les champs « résumé isomorphe » et « commentaires»), ton général. Ces données seront également mises à contribution pour l'autre volet du projet, qui porte sur la vie culturelle montréalaise. Les assistants de recherche qui colligeaient les données réalisaient en outre des rapports de recherche visant à caractériser la couverture de chacun des journaux et à dégager des pistes de réflexions. À cette étape d'accumulation de données, l'équipe n'était ni à la recherche de textes « intéressants », ni dans une logique de problématisation. C'est le croisement des données rendu possible par l'interrogation de la banque qui a nourri le développement des problématiques et permis 
d'identifier certains phénomènes. Par exemple, lors du croisement des données, la grande quantité d'éléments usant de la première personne du pluriel a incité les rédacteurs de l'article «Les Jeux olympiques de Berlin dans l'arène médiatique montréalaise " à intégrer à ses travaux la question de la nation, qui s'est finalement révélée riche et complexement organisée, même si le «nation-building canadien" est postérieur (rappelons que le Canada devient véritablement indépendant de la Grande-Bretagne seulement en 1931) et si l'emploi d'expressions comme "nos athlètes ", "nos équipes ", paraissaient aller de soi et constituaient de ce fait le point aveugle des rapports. Le travail s'est en somme fait en quatre étapes distinctes, enrichies par de nombreuses discussions : l'analyse de tous les éléments du journal et la saisie des données en résultant; la rédaction de rapports de recherche individuels sur chacun des périodiques; l'extraction des éléments ayant les JO comme objet; le croisement des divers résultats de l'analyse grâce aux interrogations de la banque suscitées par les pistes identifiées dans les rapports de recherche, par les effets de masse repérés dans la banque ou par les échanges au sein de l'équipe ou avec les membres des autres équipes. Cette dernière étape, passionnante, a été effectuée de manière collégiale : la présence d'expressions récurrentes identifiables grâce au « résumé isomorphe » a favorisé le développement d'une perspective sémiotique ancrée dans la matérialité des textes.

10 L'intérêt de la banque de données, conçue comme interface de saisie et d'interrogation, a été de permettre une analyse uniforme des divers types de données, même lorsque leur valeur heuristique n'avait pas encore été établie. La caractérisation médiatique des éléments à partir de descriptions uniformes et fiables, évitait de retourner à tout moment aux journaux ou, au contraire, incitait à le faire puisqu'elle permettait de distinguer les éléments les plus riches. La possibilité de croiser rapidement les données, afin par exemple de repérer les éléments qui portaient à la fois sur les JO et sur la politique, a permis de donner une profondeur statistique au travail tout en favorisant l'évolution de la problématisation et la mise en jeu des hypothèses.

11 Il faut ajouter que la rencontre internationale tenue en mai 2014 a eu une portée considérable sur les travaux, faisant découvrir à l'équipe, par contraste, la pauvreté de la couverture montréalaise des Jeux, principalement issue des communiqués des agences de presse. Aucun journaliste montréalais n'a été envoyé par sa rédaction à Berlin, aucun n'a été accrédité par le CIO comme le révèlent les listes d'accréditations qui ont été communiquées à tous par l'équipe suisse. En revanche, la façon d'aborder les questions de race et de genre s'est révélée originale, ce qui a mené à la décision de rédiger un article développant cet aspect, et un bref article sur Myrtle Cook.

\section{L'équipe de Montpellier}

12 L'équipe de Montpellier se composait de chercheurs (Amélie Chabrier, Marie-Ève Thérenty, et Yoan Vérilhac), de doctorants (Laure Demougin, Héléna Demerdjian, Filippos Katsanos, Malek Khbou, Mélodie Simard-Houde) et de stagiaires de recherche rassemblés dans le contexte d'un centre de recherche, le RIRRA21, laboratoire en littérature et arts de l'université de Montpellier III. Le travail de dépouillement s'est effectué à partir de périodiques dont certains étaient numérisés sur la plate-forme Gallica, parfois en mode texte, ce qui permettait une recherche par mots-clés. Certains journaux, notamment des hebdomadaires, ont été achetés ; enfin d'autres encore ont été consultés en bibliothèque, à la Bibliothèque municipale de Lyon ou à la Bibliothèque nationale de France. Très 
rapidement il est apparu que l'utilisation de la base de données canadienne de dépouillement serait difficile. D'abord les journaux français contenaient une abondance de nouvelles très chronophages à répertorier dans un contexte universitaire et scientifique peu propice au dépouillement de longue durée (les centres de recherche français, à la différence du système canadien, ne disposent pas d'assistants de recherche rémunérés et ce manque a des conséquences essentielles sur les modalités et les formes de la recherche). Ensuite, l'équipe montpelliéraine, du fait de son histoire et de sa culture, était plus particulièrement formée pour étudier les questions de poétique historique et notamment l'hybridation entre la matrice littéraire et la matrice médiatique. Ce qui l'intéressait tout particulièrement dans ce projet, c'était d'une part de reprendre et peutêtre amender l'idée d'une rupture nette entre les poétiques médiatiques du XIX ${ }^{\mathrm{e}}$ siècle et celles de l'entre-deux-guerres et d'autre part, plus globalement, d'interroger par sondages, les modalités d'écriture et de rendu du réel de la presse des années trente condamnée à la Libération pour sa tendance à la calomnie et à la diffamation. Il semblait nécessaire de revenir peut-être de manière très pragmatique à la fabrication de l'information et à l'investigation du rapport vérité-fiction pour déterminer si le rapport perverti au vrai impliqué dans la calomnie ne devait pas s'interpréter comme un élément relevant d'un système global d'information.

13 L'équipe montpelliéraine s'est d'abord nourrie de la lecture des trois livres relativement récents qui en France, sont revenus sur l'histoire des Jeux: Fabrice Abgrall et François Thomazeau, La France à l'épreuve des Jeux Olympiques de Berlin, Alvik éditions, 2006 ; JeanMarie Brohm, 1936, Les Jeux olympiques à Berlin, André Versaille éditeur, 2008 ; Jean-Marie Blaizeau, Les Jeux défigurés, Les Indes Savantes, 2012. Il est à noter que ce dernier ouvrage reprend le titre d'un éditorial de L'Auto, le principal journal sportif de l'époque. Il est d'ailleurs préfacé par l'éditorialiste de L'Auto, Jacques Goddet, futur directeur de L'Équipe et il véhicule une lecture de L'Auto qui sans être complètement fausse occulte certaines de ses positions pendant l'olympiade. À cette référence près, ces ouvrages ne revenaient qu'imprécisément sur la manière dont les journaux français avaient rendu compte de l'olympiade mais se fondaient beaucoup plus sur les débats d'avant 1936, à propos d'un éventuel boycott, qui effectivement avaient été très clivants dans la presse. L'équipe montpelliéraine a ensuite fixé son corpus de dépouillement. Compte tenu du grand nombre de journaux, il était impossible de viser l'exhaustivité. L'équipe de Montpellier a donc dépouillé quatre types de périodiques : des quotidiens d'information (reportage) et d'opinion (Le Petit Parisien, Le Matin, Le Petit Journal, Le Figaro, L'Écho de Paris, le Temps, L'Action française, L'Euvre, L'Humanité, Paris-Soir, L'Intransigeant, La Croix, Le Progrès), des hebdomadaires et notamment les nouvelles formules des magazines d'actualité (Lectures pour tous, Je sais tout, Candide, Gringoire, Vendredi, Je suis partout, Voilà, Vu, Marianne et L'Illustration), des journaux sportifs (L'Auto, Match L'Intran, Le Miroir des sports) et de la presse satirique (Le Canard enchaîné et Le Charivari). À partir des premiers dépouillements, l'équipe française a repéré une série de motifs qui apparaissent comme des nœuds axiologiques ou politiques et qui permettent d'identifier les positions idéologiques et polémiques des journaux. Elle a sérié cinq moments climatériques qui sont systématiquement l'objet de développements axiologiques : la mise en scène nazie et la propagande, le salut de Joinville fait par l'équipe française et identifié par le public allemand du stade comme un salut nazi, la victoire de Jesse Owens, l'olympisme, les pitoyables résultats des sportifs français. À partir de l'observation de ces motifs et du fonctionnement poétique de l'ensemble de la rubrique sportive, l'équipe a mis au point 
une fiche analytique divisée en deux rubriques : «la fabrique de l'événement sportif » et "l'interaction entre sport et idéologie, " et comportant une grille de points à sonder. Cette fiche a constitué le squelette commun de tous les dépouillements et a permis à des chercheurs novices dans le domaine de la presse d'être immédiatement sensibles à des questions de rubriques, à la hiérarchie et à l'étoilage de l'information sportive (les JO peuvent être évoqués à la rubrique sportive mais aussi dans d'autres rubriques, par exemple politiques), à des interrogations sur les rédacteurs des articles (s'agissait-il de journalistes connus pour leurs plumes, d'experts auteurs de compte rendus plus techniques, de sportifs en activité ou reconvertis dans le journalisme?) ou à des phénomènes stylistiques (ellipses) ou énonciatifs. Toutes les fiches ont été stockées sur un nuage commun de données, accessible à tous. L'équipe s'est réunie de manière régulière lors de séminaires qui permettaient de suivre la progression des dépouillements et de discuter collectivement la pertinence et la généralisation possible des nouvelles découvertes. L'article présenté par l'équipe française a été rédigé collectivement, grâce à un logiciel qui permettait de partager les données. L'équipe a aussi décidé de la rédaction de deux articles particuliers portant l'un sur la question cruciale du traitement photographique des Jeux, l'autre sur la figure très internationale et un peu fantasque de Mrs Eleanor Holm Jarret.

\section{L'équipe de Bruxelles}

14 Composée de deux chercheurs, Paul Aron et Vanessa Gemis, appuyée d'un groupe de six étudiants rassemblés dans le cadre d'un séminaire dont la tenue s'est étalée sur trois mois, l'équipe de Bruxelles a souhaité doubler le travail de recherche d'une réflexion sur l'intérêt pédagogique de l'étude de presse pour des étudiants en lettres. Il s'agissait de montrer concrètement à ces derniers les échanges entre vie littéraire et médiatique, y compris dans les techniques d'écriture et les genres, mais également de leur prouver que leur formation leur permettait de LIRE le journal d'une manière sinon novatrice, du moins intéressante. Deux axes d'analyse ont guidé l'encadrement pédagogique :

$151^{\circ}$ la notion d'intermédialité : en s'intéressant plus spécifiquement à la forme du reportage, les étudiants ont été invités à interroger la porosité des frontières entre presse et littérature, à la fois en termes de poétique discursive et de poétique du support. Les séances théoriques (cf. infra) ont par ailleurs largement encouragé une lecture intermédiale (projection d'un documentaire d'époque, présentation de différents types de documents, accent mis sur la poétique du support médiatique, etc.).

$162^{\circ}$ les notions d'engagement et d'idéologie en littérature : il s'agissait d'interroger les liens entre le politique et le littéraire, en s'intéressant en particulier à l'écriture journalistique - ici le reportage sportif - en registre contraint. Les étudiants ont en particulier été invités à lier leurs analyses textuelles aux enjeux extra-littéraires spécifiques à leur corpus.

17 Deux séances «théoriques » ont permis de baliser la recherche. La première séance fut spécifiquement consacrée aux JO de 1936. Le documentaire de la réalisatrice allemande Leni Riefenstahl, Les Dieux du Stade (1938) ainsi que de nombreux documents d'époque (plan du site olympique, affiches de propagande, ouvrages commémoratifs, etc.) ont permis d'amorcer la réflexion sur les enjeux des Jeux et d'émettre les premières hypothèses de recherche. La seconde séance s'est davantage centrée sur le champ de la recherche consacrée aux rapports entre presse et littérature. Il s'agissait ici de 
sensibiliser les étudiants aux différents niveaux de lecture du journal (visuel, textuel, historique). Ces deux séances ont également été l'occasion de pointer les sources critiques indispensables à l'analyse.

18 Le corpus des périodiques à travailler a été prédéfini en amont par P. Aron et V. Gemis, tout comme les «sujets" - deux à trois titres de presse à confronter - proposés aux étudiants. Ces journaux s'inscrivant eux-mêmes dans des courants idéologiques distincts, les étudiants ont été invités à documenter leur corpus à l'aide des sources critiques (articles, monographies, etc.) consacrées à la presse belge. Ce volet du travail a permis aux étudiants de découvrir les ressources consacrées à la presse belge et les politiques de conservation des bibliothèques nationale et universitaires. Les journaux retenus étaient en effet disponibles sous divers formats (papier, microfilmé, numérique), et ont donc nécessité des traitements distincts. Seuls les journaux numérisés ont permis une recherche plein texte, et dès lors de déborder du cadre chronologique de la tenue des Jeux, pour s'intéresser aux débats préalables et conclusifs. Ce mode de recherche a également permis de situer l'intérêt porté par la presse aux Jeux dans le flux médiatique de l'époque.

Destiné aux étudiants de troisième bachelier en langues et lettres françaises et romanes, le séminaire avait également pour ambition d'initier à la recherche en littérature et à la rédaction d'un travail scientifique. Il s'agissait donc tout à la fois d'offrir des cadres d'analyse et de garantir aux étudiants un espace de réflexion et d'apprentissage. Hormis les deux séances introductives, le séminaire s'est résolument construit en dialogue avec les étudiants. Une étape de présentation orale a en particulier permis d'affiner collégialement les hypothèses de recherches et de les confronter aux pistes dégagées individuellement. L'analyse du corpus belge proposée par Paul Aron et Vanessa Gemis s'est donc développée à partir des travaux présentés par les étudiants. Les pistes dégagées par ces derniers ont fait l'objet d'un approfondissement d'où il a été possible de dégager certaines spécificités locales.

20 L'expérience pédagogique menée par l'équipe de Bruxelles s'inscrit dans un contexte plus général de rapprochement entre les filières de langues et lettres, et d'information et de communication au sein de l'Université libre de Bruxelles. Elle participe par ailleurs d'une volonté d'offrir aux étudiants en lettres des débouchés professionnels dans les champs médiatiques, en leur assurant une formation à l'analyse et l'écriture journalistique. Plusieurs cours et séminaires ont ainsi été proposés ces dernières années sur les liens entre littérature et journalisme, certains misant directement sur une collaboration entre les deux filières (cours et travaux communs, invitations de professeurs, etc.). L'intérêt des étudiants inscrits en langues et littératures romanes pour ces questions peut à la fois se mesurer à leur investissement - on peut même parler d'enthousiasme - dans un séminaire tel que celui consacré au traitement médiatique des Jeux olympiques de Berlin, mais également au nombre croissant de travaux de maîtrise consacrés à ces questions.

\section{L'« équipe » de Lausanne}

21 Plutôt qu'une équipe, il s'agit ici d'un tandem constitué de deux historiens spécialistes de l'histoire des médias. N'ayant pu intégrer cette démarche dans un projet d'enseignement ou de recherche plus large, les protagonistes ont procédé eux-mêmes au dépouillement des journaux suisses en profitant de la numérisation avancée d'un bon nombre de quotidiens. Afin de garantir une représentativité maximale des supports privilégiés, des 
titres plus spécifiques ont été pris en compte : on songe notamment à la presse socialiste, encore peu numérisée au moment de l'enquête, à la presse illustrée ainsi qu'à plusieurs journaux sportifs. Par rapport aux autres équipes internationales, la focale chronologique a été élargie aux mois qui précèdent la manifestation berlinoise. En effet, le mouvement de boycott donne lieu en Suisse à un important débat public dès l'été 1935. Par ailleurs, il a paru intéressant de souligner combien le traitement de l'événement est intimement lié à un contexte plus large tant politique, diplomatique que sportif : en ce sens, les enjeux de politique intérieure, les relations avec le nouveau régime nazi ou les échos des Jeux d'hiver de Garmisch sont essentiels à prendre en compte. Enfin, les deux chercheurs ont $\mathrm{pu}$ mener quelques investigations ponctuelles au sein des archives du Comité international olympique sises à Lausanne. Celles-ci donnent des informations importantes sur le dispositif médiatique de la manifestation ainsi que sur les coulisses de l'organisation. Au final, la confrontation des « narrations berlinoises » tant au niveau des orientations idéologiques des journaux que de la diversité générique des supports révèle un traitement plus diversifié que le portrait donné par la littérature secondaire existante sur le sujet. En marge du récit des compétitions et de l'énumération fastidieuse des résultats, nombreux sont les commentaires jouant avec une forte part d'implicite ou maniant l'euphémisme, voire l'ironie.

\section{Conclusions}

\section{Quelques limites du projet}

Les interactions entre presse et radio constituent l'un des points aveugles de la recherche. En effet, la cérémonie d'ouverture des Jeux de Berlin est radiodiffusée, grâce à une technologie nouvelle et complexe. Certains journaux montréalais en rendent compte ${ }^{4}$. Mais nous savons peu de choses de la manière dont la radio s'était emparée de l'événement des JO. Cela tient-il à une forme de concurrence entre médias? Les textes n'en parlent guère. Le travail en cours de l'équipe internationale sur l'Affaire Lindbergh invite à penser qu'il existait peut-être une convention quant à la manière de renvoyer à des sources radiophoniques : mention d'une ville seule, sans signature ni mention d'un service de presse. Mais cela demeure une hypothèse. Nous devons avouer que nous ne savons pas dans quelle mesure la radio a contribué à diffuser les résultats des JO ou à répercuter, à partir de l'événement, les débats socio-politiques afférents dans l'espace public.

23 L'une des caractéristiques de notre travail tient à la variété des systèmes de presse étudiés. Cela constitue à la fois une limite et un atout. Ainsi la France dispose de périodiques exclusivement réservés au sport, ce qui explique sans doute en partie le grand nombre de correspondants envoyés à Berlin, tandis que les journaux montréalais, généralistes (aucun périodique n'est consacré au sport en ces années) n'ont délégué personne. Comparant la manière dont les divers sujets sont traités, nous comparons des systèmes fort diversement organisés dans lesquels il est tentant de voir des lacunes ou des genres prédominants, selon les cas. Néanmoins, au fil des discussions, cette variété a permis à l'équipe élargie d'interroger autrement les pratiques, par exemple le genre du reportage. Absent ou presque dans les journaux montréalais, abondant voire omniprésent dans les journaux français et belges, surtout présent en Suisse dans les journaux illustrés, grâce au travail de photographes qui ne font pas partie des agences allemandes, ou dans 
les rares fragments subjectifs qui introduisent les comptes rendus des résultats dans la grande presse, le reportage se révèle, dans une perspective macro, pourrait-on dire, en concurrence avec d'autres formes dans lesquelles s'exprime la subjectivité du signataire. Les chroniques des journaux montréalais apparaissent ainsi comme le principal lieu d'exercice de la subjectivité en ce qui a trait aux Jeux. Lieu des mises en contexte politique, des jugements moraux, des interrogations, ces derniers agissent comme structure de cadrage ( framing $^{5} »$ ) des informations. Ils réduisent la dispersion de l'information qui les jouxte et introduisent une forme de cohérence. Au contraire, dans le reportage tel que pratiqué en France ou en Belgique, subjectivité et objectivité s'entremêlent et s'appuient l'une sur l'autre, créant un « réel » séduisant et convaincant, mais totalisant. Bref, l'examen des pratiques, par-delà la diversité des systèmes de presse, invite à travailler autrement sur les formes en rappelant l'importance de leur dimension pragmatique.

\section{Une certaine façon de lire}

Les résultats des travaux, effectués dans des conditions variées et dans le contexte de préoccupations de recherche différentes, ont révélé la forte cohérence méthodologique de nos travaux respectifs, par delà la différence des protocoles de recherche. En effet, la perspective littéraire, appuyée sur les postulats développés dans la théorie du discours social de Marc Angenot et des tenants de la sociocritique transforme profondément le travail sur la presse, et sur les textes. Celui-ci est porté par un double regard, à la fois éloigné, attentif à l'interdiscusivité, aux régularités narratives et sémiotiques, à la mosaïque générale dans laquelle chacun d'eux s'insère, et rapproché, c'est-à-dire attentif à la matérialité du support et à ses effets stylistiques, aux tensions liées aux effets de signature, aux récurrences et aux trous, à l'expression concrète d'une poétique des genres.

En somme nous avons, sans que cela soit prémédité, travaillé de la même façon, Cela indique que, depuis la fin des années 1970, s'est graduellement développée une manière de lire le journal dont divers travaux ont constitué des jalons ${ }^{6}$. Cette manière littéraire de lire le journal constitue désormais un acquis apte à faire l'objet de protocoles adaptés à des objets différents sans que la perspective commune ne soit affectée.

Cette manière revêt à nos yeux une remarquable fécondité pédagogique. Elle enseigne aussi à lire autrement, par une prise en compte, dans l'ordre de l'interprétation, de la matérialité du support, par une saisie globale de la situation de l'objet qui est tout autre chose que l'examen machinal du contexte, par une prise en compte enfin de tous les aspects de l'énonciation, saisis comme dynamiques. Le journal apparait ainsi comme un adjuvant dans le développement d'outils fins de lecture pour les étudiants qui acceptent de jouer le jeu de cette matière textuelle trop souvent considérée comme l'autre de la littérature. 


\section{NOTES}

1. Fernand Dumont écrit «Si le journal m'apporte de l'« information », comme on dit, ce n'est pas parce qu'il est le reflet du monde, mais parce qu'il est un objet inséré entre ma conscience et le monde et que, par là, il devient générateur d'un autre monde " (Le lieu de l'homme, Montréal, $\mathrm{BQ}$, 1994, p. 78 [1968]).

2. D'autres chercheurs de l'équipe " presse montréalaise » ont collaboré à la mise en forme des outils de recherche et suivi les travaux de dépouillement sans y participer: Marie-Josée Des Rivières, Pierre Anctil, Denis Saint-Jacques et Will Straw.

3. Les travaux de Jean de Bonville sur la presse du XIX ${ }^{\mathrm{e}}$ siècle se terminent en 1914 (Jean de Bonville, La Presse québécoise de 1884 à 1914 : genèse d'un média de masse, Québec, Presses de l'Université Laval, 1988) et ceux sur la période moderne commencent après la seconde guerre (Jean de Bonville, Les Quotidiens montréalais de 1945 à 1985 : morphologie et contenu, Québec, Institut québécois de recherche sur la culture, 1995).

4. Un article du numéro de la semaine du 2 au 8 août 1936 de l'hebdomadaire suisse Le Radio porte spécifiquement sur le dispositif radiophonique de la diffusion de la cérémonie d'ouverture.

5. Dennis Chong et James N. Druckman. « Framing theory », Annual Review of Political Science, Vol. 10, 2007, p. 103-126.

6. "Annexe. Questions de méthode", Marie-Ève Thérenty et Alain Vaillant, L'an un de l'ère médiatique. Analyse liittéraire et historique de La Presse de Girardin, Paris, Nouveau monde éditions, 2001, p. [297]-360; Micheline Cambron, « De l'importance de la facture des périodiques dans la compréhension de l'histoire de la littérature du Bas-Canada », Fac-Simile, n 14, novembre 1995, p. 12-15 (Parution simultanée en anglais sous le titre " The Importance of Periodical Format and Styles : Understanding the History of Literature in Lower Canada ») et Micheline Cambron (dir.), Le Journal Le Canadien. Littérature, espace public et utopie (1836-1845) (dir.), Montréal, Fides, coll. "Nouvelles études québécoises », 1999, 424 p. Sans oublier le monumental ouvrage La civilisation du journal. Histoire culturelle et littéraire de la presse française au XIXe siècle, Dominique Kalifa, Philippe Régnier, Marie-Ève Thérenty et Alain Vaillant (dir.), Paris, Nouveau monde éditions, 2011,1762 p.

\section{ABSTRACTS}

Cet article revient sur la méthodologie suivie par les différentes équipes nationales, nécessairement différente en raison de la disparité des conditions de la recherche. Il insiste néanmoins sur le savoir partagé qui a été mobilisé, qui tient à des interrogations communes sur la poétique du support et à l'intérêt d'étudier en profondeur les modes d'écriture de la presse. 


\section{INDEX}

Mots-clés: Presse, sociocritique, poétique des genres, Jeux olympiques, poétique du support, énonciation, pédagogie, lecture

\section{AUTHORS}

\section{PAUL ARON}

Paul Aron est directeur de recherches au FNRS et professeur à l'ULB. Il est l'auteur de nombreux travaux en sociologie et en histoire de la littérature de langue française et de travaux sur les liens entre presse et littérature, notamment en Belgique francophone.

\section{MICHELINE CAMBRON}

Micheline Cambron est professeure au Département des littératures de langue française de l'Université de Montréal et membre du Centre de recherche interuniversitaire sur la littérature et la culture québécoise (CRILCQ. Elle consacre ses recherches et son enseignement à la littérature et à la culture québécoises des $\mathrm{XIX}^{\mathrm{e}}$ et $\mathrm{Xx}^{\mathrm{e}}$ siècles. Elle a principalement travaillé sur les formes de l'utopie, les relations presse/littérature et les questions d'histoire littéraire et culturelle. Elle est également spécialiste des œuvres de Fernand Dumont et Paul Ricœur.

\section{GIANNI HAVER}

Gianni Haver est professeur associé de sociologie de l'image et d'histoire sociale des médias à l'Université de Lausanne. Il s'intéresse particulièrement aux productions médiatiques de la période de l'entre-deux-guerres et de la Deuxième Guerre mondiale et aux rapports entre histoire et médias. Il a notamment travaillé sur le cinéma et, plus récemment, sur les comics et la photo de presse. Il dirige la collection « Médias et histoire » aux éditions Antipodes.

\section{MARIE-ÈVE THÉRENTY}

Marie-Ève Thérenty est professeur de littérature française et directrice de l'équipe de recherche RIRRA21 de l'université Paul-Valéry Montpellier 3. Elle codirige avec Guillaume Pinson le site medias19.org. Elle est l'auteur de nombreux articles et livres sur les rapports entre littérature et médias, sur la poétique des supports et sur l'imaginaire médiatique des sociétés.

\section{FRANÇOIS VALLOTTON}

François Vallotton est professeur ordinaire d'histoire contemporaine à l'Université de Lausanne. Auteur de nombreuses contributions sur l'histoire culturelle et intellectuelle, il a consacré de nombreux ouvrages et articles à l'histoire du livre et de l'édition, à l'histoire de la presse ainsi qu'à l'histoire de la radio et de la télévision, dans une perspective suisse mais aussi transnationale. Il est également membre fondateur du Centre interdisciplinaire des Sciences historiques de la culture (Université de Lausanne). 